SECTION 2. Applied mathematics. Mathematical modeling.

\author{
Naumov Anatoly Aleksandrovich \\ candidate of technical Sciences, Associate Professor, \\ Center of Applied Mathematical Research, Novosibirsk, Russia \\ E-mail: A_A_Naumov@mail.ru
}

\title{
TO THE PROBLEMS OF NORMALIZATION METHOD OF CRITERIA IN PROBLEMS OF VECTOR OPTIMIZATION
}

In this work the features of the method of normalization of the criteria applied to problem of multicriteria (vector) optimisation. Shows the weaknesses of the method, which lead to the conclusion that, with regard to economic challenges the use of this method is not appropriate.

Keywords: vector optimization, criterion, economic task.

\section{К ПРОБЛЕМАМ МЕТОДА НОРМАЛИЗАЦИИ КРИТЕРИЕВ В ЗАДАЧАХ ВЕКТОРНОЙ ОПТИМИЗАЦИИ}

В работе исследованы особенности метода нормализации критериев применительно к задаче многокритериальной (векторной) оптимизации. Показаны слабые места метода, которые приводят к выводу, что применительно к экономическим задачам использование данного метода представляется нецелесообразным.

Ключевые слова: векторная оптимизаиия, критерий, экономическая задача.

В работе исследованы особенности метода нормализации критериев (см. [1], [2]) применительно к задаче многокритериальной (векторной) оптимизации.

Постановка задачи. Предположим, что необходимо решить многокритериальную задачу линейного программирования:

$$
\begin{gathered}
\mathrm{f}_{1}\left(\mathrm{x}_{1}, \mathrm{x}_{2}, \mathrm{x}_{3}, \ldots, \mathrm{x}_{\mathrm{n}}\right) \rightarrow \max \\
\mathrm{f}_{2}\left(\mathrm{x}_{1}, \mathrm{x}_{2}, \mathrm{x}_{3}, \ldots, \mathrm{x}_{\mathrm{n}}\right) \rightarrow \max \\
\ldots \\
\mathrm{f}_{\mathrm{p}}\left(\mathrm{x}_{1}, \mathrm{x}_{2}, \mathrm{x}_{3}, \ldots, \mathrm{x}_{\mathrm{n}}\right) \rightarrow \max
\end{gathered}
$$

при ограничениях:

$$
\left\{\begin{array}{c}
g_{1}\left(x_{1}, x_{2}, x_{3}, \ldots, x_{n}\right) \leq b_{1} \\
g_{2}\left(x_{1}, x_{2}, x_{3}, \ldots, x_{n}\right) \leq b_{2} \\
\ldots \\
g_{m}\left(x_{1}, x_{2}, x_{3}, \ldots, x_{n}\right) \leq b_{m}
\end{array}\right.
$$


Задача линейного программирования была выбрана для упрощения рассуждений. Предположим, что область допустимых решений задачи (обозначим ее через D), образованная ограничениями (2), является непустой, выпуклой и ограниченной. Таким образом, на этой области существуют оптимальные решения для каждого из критериев множества (1).

Основная идея метода нормализации критериев состоит в переходе от многокритериальной задачи (1)-(2) к скалярной задаче ([1], [2]). Для этого сначала критерии (1) нормализуют в соответствии с формальными записями:

$$
\begin{array}{r}
\lambda_{1}\left(\mathrm{x}_{1}, \mathrm{x}_{2}, \mathrm{x}_{3}, \ldots, \mathrm{x}_{\mathrm{n}}\right)=\frac{\mathrm{f}_{1}\left(\mathrm{x}_{1}, \mathrm{x}_{2}, \mathrm{x}_{3}, \ldots, \mathrm{x}_{\mathrm{n}}\right)-\mathrm{f}_{1}^{\min }}{\mathrm{f}_{1}^{\max }-\mathrm{f}_{1}^{\min }} \\
\lambda_{2}\left(\mathrm{x}_{1}, \mathrm{x}_{2}, \mathrm{x}_{3}, \ldots, \mathrm{x}_{\mathrm{n}}\right)=\frac{\mathrm{f}_{2}\left(\mathrm{x}_{1}, \mathrm{x}_{2}, \mathrm{x}_{3}, \ldots, \mathrm{x}_{\mathrm{n}}\right)-\mathrm{f}_{2}^{\min }}{\mathrm{f}_{2}^{\max }-\mathrm{f}_{2}^{\min }}, \\
\ldots \\
\lambda_{\mathrm{p}}\left(\mathrm{x}_{1}, \mathrm{x}_{2}, \mathrm{x}_{3}, \ldots, \mathrm{x}_{\mathrm{n}}\right)=\frac{\mathrm{f}_{\mathrm{p}}\left(\mathrm{x}_{1}, \mathrm{x}_{2}, \mathrm{x}_{3}, \ldots, \mathrm{x}_{\mathrm{n}}\right)-\mathrm{f}_{\mathrm{p}}^{\min }}{\mathrm{f}_{\mathrm{p}}^{\max }-\mathrm{f}_{\mathrm{p}}^{\min }},
\end{array}
$$

а затем формируют скалярную максиминную задачу вида:

$$
\lambda \rightarrow \max ,
$$

при ограничениях:

где

$$
\left\{\begin{array}{c}
\lambda-\lambda_{1}\left(x_{1}, x_{2}, x_{3}, \ldots, x_{n}\right) \leq 0 \\
\lambda-\lambda_{2}\left(x_{1}, x_{2}, x_{3}, \ldots, x_{n}\right) \leq 0 \\
\ldots \\
\lambda-\lambda_{p}\left(x_{1}, x_{2}, x_{3}, \ldots, x_{n}\right) \leq 0 \\
g_{1}\left(x_{1}, x_{2}, x_{3}, \ldots, x_{n}\right) \leq b_{1} \\
g_{2}\left(x_{1}, x_{2}, x_{3}, \ldots, x_{n}\right) \leq b_{2} \\
\ldots \\
g_{m}\left(x_{1}, x_{2}, x_{3}, \ldots, x_{n}\right) \leq b_{m}
\end{array}\right.
$$

$\lambda=\min \left(\lambda_{1}\left(\mathrm{x}_{1}, \mathrm{x}_{2}, \mathrm{x}_{3}, \ldots, \mathrm{x}_{\mathrm{n}}\right), \lambda_{2}\left(\mathrm{x}_{1}, \mathrm{x}_{2}, \mathrm{x}_{3}, \ldots, \mathrm{x}_{\mathrm{n}}\right), \ldots, \lambda_{\mathrm{p}}\left(\mathrm{x}_{1}, \mathrm{x}_{2}, \mathrm{x}_{3}, \ldots, \mathrm{x}_{\mathrm{n}}\right)\right) ;$

$\mathrm{f}_{\mathrm{i}}^{\min }, \mathrm{f}_{\mathrm{i}}^{\max }, \mathrm{i}=1,2,3, \ldots, \mathrm{p}$, - это наименьшие и наибольшие значения соответствующих критериев из множества (1) на области допустимых решений (2). Эти значения получаются в результате решения 2 р скалярных оптимизационных задач. Таким образом, нормализация критериев - это некоторый искусственный прием, сводящий задачу векторной оптимизации (с множеством критериев) к скалярной задаче (с одним критерием). При этом в данном случае новая задача представляет собой так называемую максиминную задачу линейного программирования.

К сожалению, у данного метода имеется множество недостатков. Рассмотрим некоторые из них. Новые (нормализованные) критерии $\left(\lambda_{\mathrm{i}}\left(\mathrm{x}_{1}, \mathrm{x}_{2}, \mathrm{x}_{3}, \ldots, \mathrm{x}_{\mathrm{n}}\right), \mathrm{i}=1,2,3, \ldots, \mathrm{p}\right)$ не имеют размерностей. Для экономических задач это означает, что происходит обезличивание целевых 
функций, сглаживание (нивелирование, затушевывание) их особенностей и т.д. Для задач экономического характера это очень важный принципиальный момент. Каждый из таких новых (нормализованных) критериев принимает значения в интервале $[0,1]$, и это не смотря на то, что каждый из исходных критериев мог принимать значения как очень большие (например, прибыль измеренная в млн. руб.), так и меньшие единицы (например, коэффициент рентабельности). В формулах для перехода $\kappa$ нормализованным значениям критериев используются величины $\mathrm{f}_{\mathrm{i}}^{\text {min }}, \mathrm{i}=1,2,3, \ldots, \mathrm{p}$, которые влияют на решение новой (максиминной) задачи, но прямого отношения к решению исходной задачи не имеют. Список недостатков метода нормализации может быть продолжен. Отсюда может быть сделан вывод. Использовать метод для эффективного решения экономических задач нежелательно, т.к. полученное при этом решение не может быть логично интерпретировано в терминах исходной задачи.

Рассмотрим иллюстративный пример. Рассмотрим две задачи с одинаковыми критериями и разными ограничениями:

$$
\begin{aligned}
& \mathrm{f}_{1}\left(\mathrm{x}_{1}, \mathrm{x}_{2}\right) \equiv 1000 \cdot \mathrm{x}_{2} \rightarrow \max , \\
& \mathrm{f}_{2}\left(\mathrm{x}_{1}, \mathrm{x}_{2}\right) \equiv 0,1 \cdot \mathrm{x}_{1} \rightarrow \max ,
\end{aligned}
$$

при ограничениях двух видов

$$
\left\{\begin{array}{l}
g_{1}\left(x_{1}, x_{2}\right) \equiv x_{1}+x_{2} \leq 2 \\
0 \leq g_{2}\left(x_{1}, x_{2}\right) \equiv x_{1} \leq 1,5 \\
0 \leq g_{3}\left(x_{1}, x_{2}\right) \equiv x_{2} \leq 1,5
\end{array}\right.
$$

и

$$
\left\{\begin{array}{c}
g_{1}\left(x_{1}, x_{2}\right) \equiv x_{1}+x_{2} \leq 2 \\
0 \leq g_{2}\left(x_{1}, x_{2}\right) \equiv x_{1} \leq 1,5 \\
0,5 \leq g_{3}\left(x_{1}, x_{2}\right) \equiv x_{2} \leq 1,5
\end{array}\right.
$$

Заметим, что области, которым принадлежат максиминные решения для задач (5)-(6) и (5)-(7) совпадают. При этом, решения этих максиминных задач различны: $x_{1}^{*}=1,0, x_{2}^{*}=1,0, f_{1}^{*}=1000, f_{2}^{*}=0,1$ (для задачи с нормализованными критериями на базе задачи (5)-(6)) и $x_{1}^{*}=0,9$, $x_{2}^{*}=1,1, f_{1}^{*}=1100, f_{2}^{*}=0,09$ (для задачи с нормализованными критериями на базе задачи (5)-(7)). Этот пример показывает зависимость решений задач с нормализованными критериями от параметров задачи, которые напрямую на области их решений (максиминных решений) не влияют.

Когда-то по поводу таких особенностей метода нормализации критериев автор этих строк пошутил так. Этот прием сродни тому, как если бы в некотором гипотетическом магазине («Гипомаркете») все товары приняли бы одинаковый внешний вид: одинаковая упаковка, одинаковые размеры, цвет, запах и т.д. Попробуйте сделать выбор необходимых Вам товаров в таком магазине! И, конечно, понятно, что рано или поздно, но 
придется переходить от решений задачи с нормализованными критериями к решениям исходной задачи. Или в примере с магазином - к исходным товарам. Заметим также, что в работе [1] авторы несколько усложнили модель. На самом деле она представляет собой оптимизационную задачу от одной переменной. Решения задачи относительно каждого отдельного критерия находятся легко вручную.

\section{Литература}

1. Кириллов Ю.В., Назимко Е.Н. Многокритериальная задача оптимизации структуры капитала и еe решение в системе Maple, Экономика и менеджмент систем управления, 2013, т. 8, № 2.1, с. 149-160.

2. Машунин Ю.К. Методы и модели векторной оптимизации. - М.: Наука, 1986. $-140 \mathrm{c}$.

3. Список трудов [Электронный ресурс]. URL: https://sites.google.com/site/ anatolynaumov2011/home/spisok-trudov-list-of-papers (дата обращения: 25.08.2013). 